\title{
Status of the KLYPVE-EUSO detector for EECR study on board the ISS
}

\author{
Pavel Klimov* \\ Lomonosov Moscow State University, Skobeltsyn Institute of Nuclear Physics (SINP MSU), \\ Moscow, Russia \\ E-mail: pavel.klimov@gmail.com \\ Marco Casolino \\ RIKEN, 2-1 Hirosawa, Wako, 351-0198 Japan \\ E-mail: casolino.marco@gmail.com
}

\section{For the JEM-EUSO Collaboration}

\begin{abstract}
KLYPVE-EUSO is a mission led by the Russian Space Agency to place an ultra-high energy cosmic ray observatory on board the Russian Segment (RS) of the ISS. The concept of the detector is based on the mirror-type detector proposed by SINP MSU in 2010 and improved by the joint studies with the JEM-EUSO collaboration. The current configuration is based on a Schmidt type optical system. This allows to increase the Field of View to 40 degrees and makes the experiment competitive with large ground-based observatories, with the advantage of the uniform exposure over the whole celestial sphere. The laboratory models of mirror-concentrator and photo detector module are being developed and manufactured. The launch of the experiment is scheduled to 2022 followed by the installation on the RS of the ISS and at least two years of operation.
\end{abstract}

35th International Cosmic Ray Conference - ICRC2017

10-20 July, 2017

Bexco, Busan, Korea

\footnotetext{
*Speaker.
} 


\section{Introduction}

The nature and origin of extreme energy cosmic rays (EECRs, those with energies $>50 \mathrm{EeV}^{1}$ ) remains one of the puzzles of modern astrophysics after more than 50 years since their first registration [1]. One of the main difficulties of the puzzle is due to the extremely low flux of EECRs. The two largest ground-based arrays (The Pierre Auger Observatory and the Telescope Array) both registered less than two dozen events with energies $\mathrm{E}>100 \mathrm{EeV}$ in 10 and 5 years of operation respectively $[2,3]$.

Nowadays, obtaining definite conclusions concerning anisotropy of EECRs becomes one of the most important tasks. As it is clear from the above, a considerable part of the difficulties in finding the sources of EECRs is due to (i) the limited statistics of events and (ii) the incomplete and non-uniform coverage of the celestial sphere by any of the modern experiments. Thus one needs to increase the statistics of EECRs providing a uniform exposition of the sky. This is exactly the field where orbital experiments open new horizons of research (not to mention a whole number of other scientific tasks related to astrophysics, fundamental physics and atmosphere sciences, which we do not discuss here) being complementary to ground-based detectors. The main idea of the method suggested by Benson and Linsley in early 1980's [4] is that fluorescent and Cherenkov radiation of an extensive air shower (EAS) generated by an EECR in the atmosphere can be detected from a satellite similar to the way it is observed from the ground but with a much larger exposure, thus considerably increasing the statistics of registered events.

The first space experiment aimed to registering EECRs is TUS [5, 6], developed in Skobeltsyn Institute of Nuclear Physics of Moscow State University (SINP MSU) in cooperation with several Russian and foreign institutions, was launched on board the Lomonosov satellite on April 28, 2016. This detector provides the first measurements of EAS candidates from orbit and demonstrates the possibility of the method.

\section{From KLYPVE to K-EUSO}

In 2012, SINP MSU finished the preliminary design stage of the KLYPVE ${ }^{2}$ telescope for EECR measurements from the International Space Station (fig. 1). It is supposed to be located on the outer side of the Russian segment of the ISS. It was designed as a large $10 \mathrm{~m}^{2}$ mirror telescope with focal distance of $3 \mathrm{~m}$ and wide field of view (FOV) - about $\pm 7.5^{\circ}[7,8]$. During the preliminary design phase it became clear that the parameters of the instrument (observation area and image quality) do not allow solve the problems related to anisotropy of EECRs.

These considerations initiated the development of a new optical system for the KLYPVE detector in order to increase the FOV and to improve the spatial and angular resolution and the overall performance of the instrument. This work began in a close cooperation with members of the JEMEUSO Collaboration in late 2013. To eliminate the off-axis aberration an additional corrective element was introduced into the telescope system in form of a Fresnel lens in front of the photodetector. Two versions of the detector were developed: Baseline and Mylti-Eye Telescope System (METS) [9, 10].

\footnotetext{
${ }^{1} 1 \mathrm{EeV}=10^{18} \mathrm{eV}$

${ }^{2}$ KLYPVE is a Russian acronym for extreme energy cosmic rays.
} 

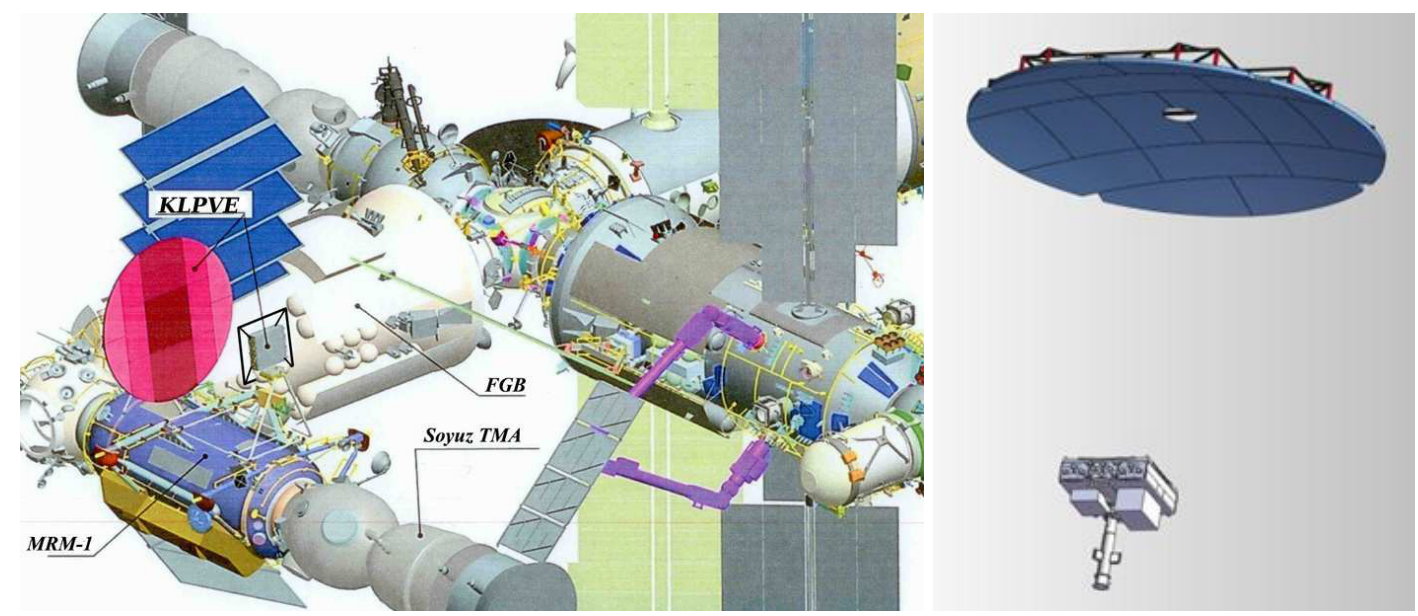

Figure 1: Left: KLYPVE on board the RS of the ISS. Right: KLYPVE segmented mirror-concentrator and photodetector.

In Baseline system (see fig. 2, left) the diameter of the reflector and the lens-corrector equals $3.4 \mathrm{~m}$ and $1.7 \mathrm{~m}$ respectively. The total length of the system is $4 \mathrm{~m}$, the distance from the lens to the focal surface equals $70 \mathrm{~cm}$. In this case, it is possible to expand the FOV up to $\pm 14^{\circ}$, and the diameter of the image is not larger than $6 \mathrm{~mm}$ in the entire FOV.

In METS option (see fig. 2, right panel) wide FOV of the detector is divided into several FOVs of identical smaller telescopes. In this case individual telescope is much simplier. In the optimization calculations, the following dimensions of an individual telescope were obtained: $2.4 \mathrm{~m}$ diameter of the mirror, $1.2 \mathrm{~m}$ lens diameter, $0.9 \mathrm{~m}$ photodetector, the total axial length $3 \mathrm{~m}$. The FOV of one telescope is $\pm 10^{\circ}$ which corresponds to the effective diameter of overall FOV of three telescopes $\sim 35^{\circ}$.

In this configuration KLYPVE-EUSO (K-EUSO) can solve definite tasks in EECR physics [11]. In Baseline design K-EUSO will detect 8000 events above $10 \mathrm{EeV}$ and over 800 events above $57 \mathrm{EeV}^{3}$ during 6 years. K-EUSO will independently confirm (or rule out) the presence of hotspots in the northern and southern hemispheres, and compare their spectral indexes.

The further development and modification of the telesope system was motivated by the following reasons:

1. Baseline desigs and METS have rather complicated aspherical shape of the mirror which make difficult its manufacturing.

2. Fresnel lenses used in this designs produce large scattering of the light and decrease the efficiency of the system.

3. TUS detector measurements demonstrate large trigger rate from the thunderstorm events out of the FOV due to scattering of the light on the roughness of the mirror. So, the system should be "closed" to be protected from the stray light.

\footnotetext{
${ }^{3}$ The energy threshold used for hotspot and anisotropy search.
} 


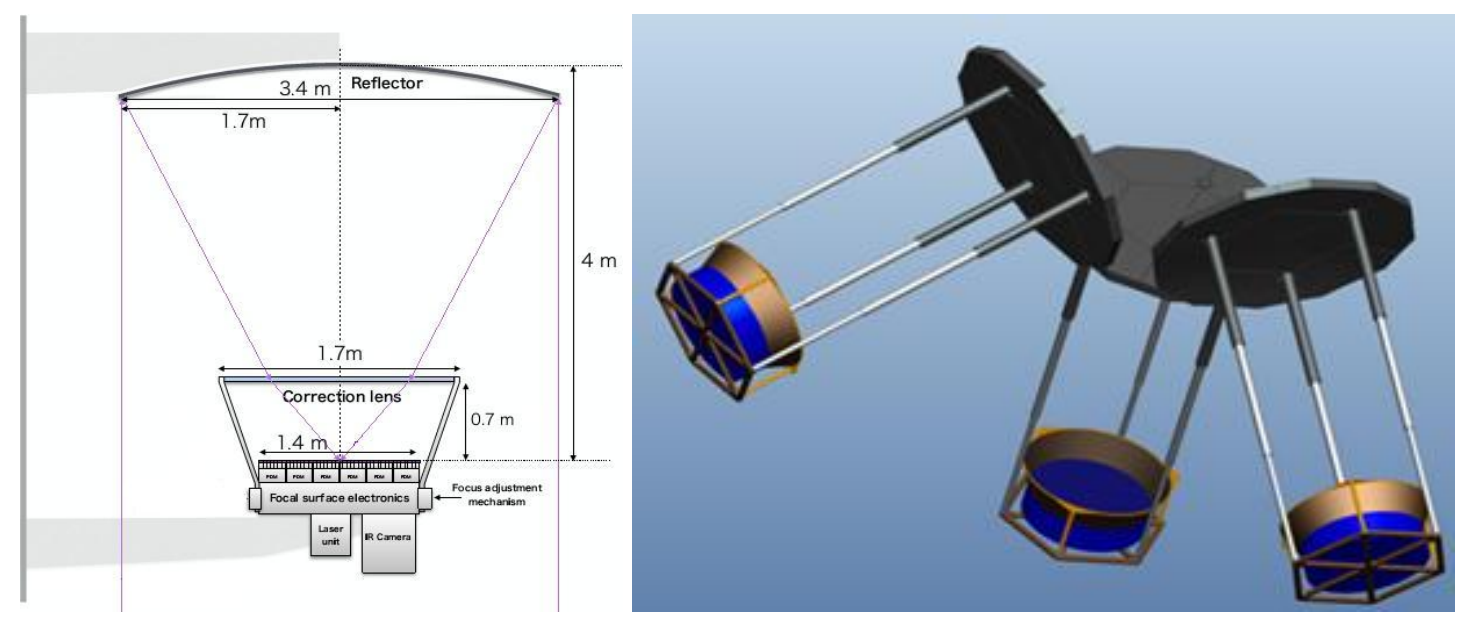

Figure 2: Left: Optics of the Baseline system. Right: optics of the METS.

4. It is desirable to increase total field of view to inlarge the EECR statistics.

A new design based on Schmidt optical system was proposed to solve all these problems. It is discribed in the next section..

\section{Schmidt telescope design for K-EUSO}

\subsection{K-EUSO optical system}

To fulfill the requirements of K-EUSO experiment, a Schmidt telescope covering a field of view of $40^{\circ}$ with an entrance pupil diameter of $2.5 \mathrm{~m}$, a $4 \mathrm{~m}$ diameter mirror and a $1.7 \mathrm{~m}$ focal length was developed. The general view of the camera is shown in fig. 3 , right. The baseline variant consists of a spherical mirror, a corrector plate and a spherical focal surface concentric with the mirror, placing the aperture stop on the frontal surface of the corrector plate. The results of the optical system simulations is presented in fig. 3, left. The very fast relative aperture (F/\# 0.7) minimizes the detector size and its obstruction, resulting in a throughput of $70 \%$ over the entire field of view. The nominal polychromatic RMS spot size is well inside the dimension of pixel size $(3 \mathrm{~mm})$ of the multi-anode PMT all over the FOV, providing an angular resolution higher than $2 \mathrm{mrad}$ (corresponding to ground resolution of about $750 \mathrm{~m}$ from the ISS orbit).

An advanced option to simplify the corrector lens shape was developed using aspherical parts on the edge of mirror. This variant can be used effectively as a deployable optics with central ( $3 \mathrm{~m}$ diameter) sperical dish and several slightly aspherical petals. In this case the profile of the corrector is much less bended at the edges, so its mass will be $66.4 \mathrm{~kg}$ only, $10 \mathrm{~kg}$ less with respect to the baseline one. For more details, see [12].

\subsection{K-EUSO photo detector}

The focal surface (FS) of the updated KLYPVE detector utilizes a design similar to that proposed for the JEM-EUSO detector [13]. It will be produced of almost 120 thousand Hama- 

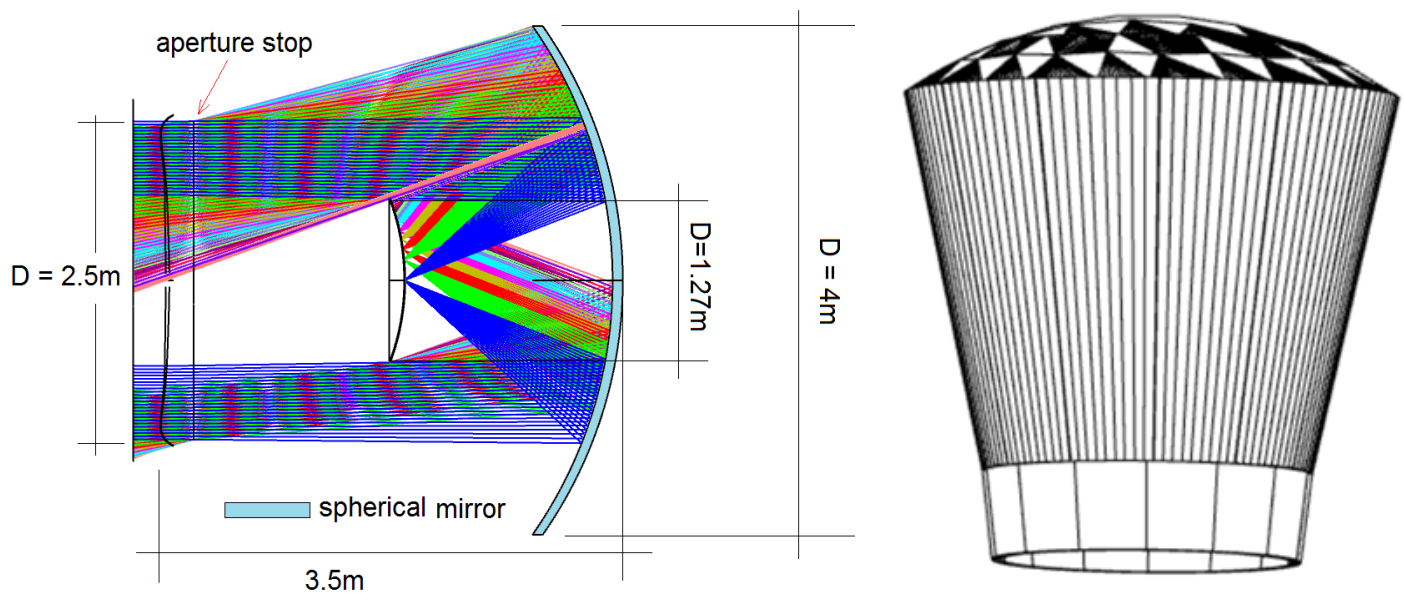

Figure 3: Left: K-EUSO Schmidt camera optics simulation results. Right:Preliminary 3D model of the K-EUSO Schmidt camera

matsu R11265-103-M64 multi-anode photomultiplier tubes (MAPMTs), grouped into photodetector modules (PDMs). The number of pixels in one MAPMT is 64, the number of MAPMTs in one PDM is 36. The size of a pixel is adjusted to the path length of an extensive air shower during one time sample (one gate time unit, $2.5 \mu \mathrm{s}$ ). The front-end electronics and high voltage power supply are the same as for the JEM-EUSO.

In the Schmidt camera design the FS has a concave shape with a diameter of $1.27 \mathrm{~m}$ and radius of curvature of $1.7 \mathrm{~m}$. There are $52 \mathrm{PDMs}$ on the FS. The JEM-EUSO concept used the hierarchical structure: 9 elementary cells composed of four PMTs are united into PDM, 8 PDMs compose a cluster, cluster of PDMs form the total FS. The hierarchical structure of the photo detector has several disadvantages. One of them is that the connection between elements possible only through the upper level that makes difficult (or even impossible) to organize cross-trigger because of very fast data flow.

In a new design each PDM realizes the entire chain of algorithms for selecting events, while in the hierarchical structure the decision about recording is taken only at the upper level of the hierarchy. All PDMs are connected to each other and united in a network. Digital part of the PDM is based on a system-on-chip solution (ZYNQ series of Xilinx). The transmission of information between the modules is carried out through communication lines. To optimize the structure of the photo detector and the interaction between its individual modules, it is proposed to use several types of connections. High-speed communication lines connect neighboring PDMs. These connections are based on the use of high-speed transceivers - dedicated terminals of the ZYNQ chip. The maximum data transfer rate for each such line is $3.125 \mathrm{~Gb} / \mathrm{s}$. Such a high transmission speed is achieved mainly due to the fact that the high-speed transceiver is connected directly to the logic cells of the FPGA. Each PDM has 8 high-speed terminals (each module is connected to all neighboring ones). Through high-speed connections, each PDM has access to information from neighboring modules, which effectively increases the field of view of each PDM by 9 times.

The second type of connection is the long-distance communication lines. These are additional links that allow improving the organization of information exchange in the network structure of the 
device. Long-distance links connect 6 specific photodetector modules via a separate switch module (fig. 4). Long-distance communications are designed to transfer information to the ROM connected to the same switch. These lines are based on the Ethernet interface.

The third type of connection is the synchronizing link. This connection is a parallel bus based on the CAN real-time protocol. The data transfer rate for this line is up to $1 \mathrm{Mb} / \mathrm{s}$. Since devices connected by this communication are switched on in parallel, any transmitted message is delivered to all devices simultaneously. This allows you to synchronize the internal clock of each PDM.

The PDM data processing (PDM DP) system is being developed and will be tested during the Mini-EUSO experiment on board the ISS in 2018. For the details see [14], these proceedings. On the fig. 5 the 3D model of current PDM design is shown (upper 3 boards are the PDM DP system).

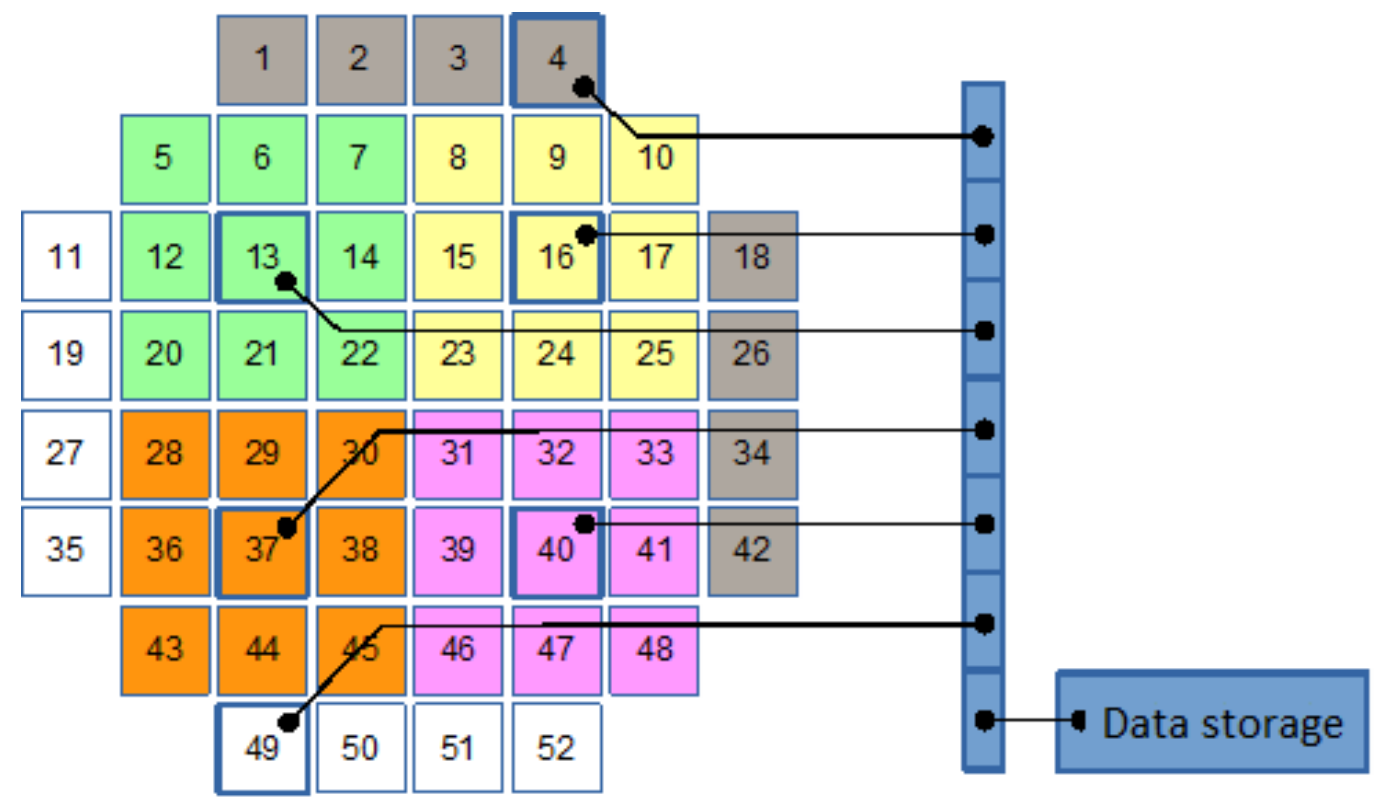

Figure 4: K-EUSO photodectector network scheme. In one color the clusters of PDMs united with switch via one communication line are shown.

\subsection{K-EUSO mechanics}

The most challenging part of the equipment design is the mechanical structure. The detector is planned to be launched with the "Progress" cargo which doesn't have the unpressurized compartment. This leads to that all parts of K-EUSO will be first stored inside the ISS and then mounted outside the station during extravehicular activity. In this case all the equipment should be divided into a number of parts whose dimensions will allow these procedures. Another option is completion of the "Progress" to avoid the transportation of the detector through the ISS windows. A sketch of the detector mechanical structure in the operation mode and during transportation is shown in fig. 6. This design allows the telescope to be transported with minimal number of the separate parts. 


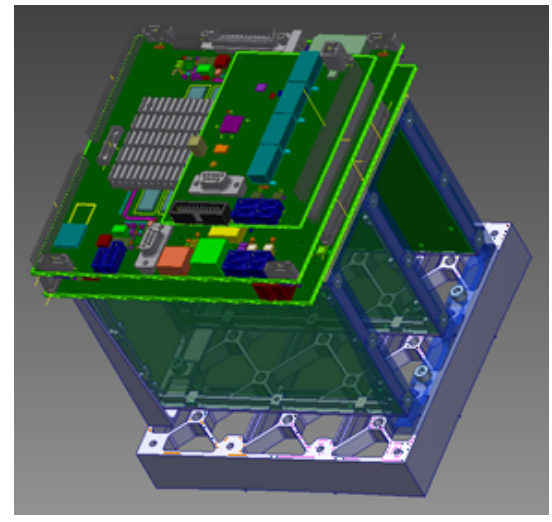

Figure 5: 3D model of K-EUSO PDM.
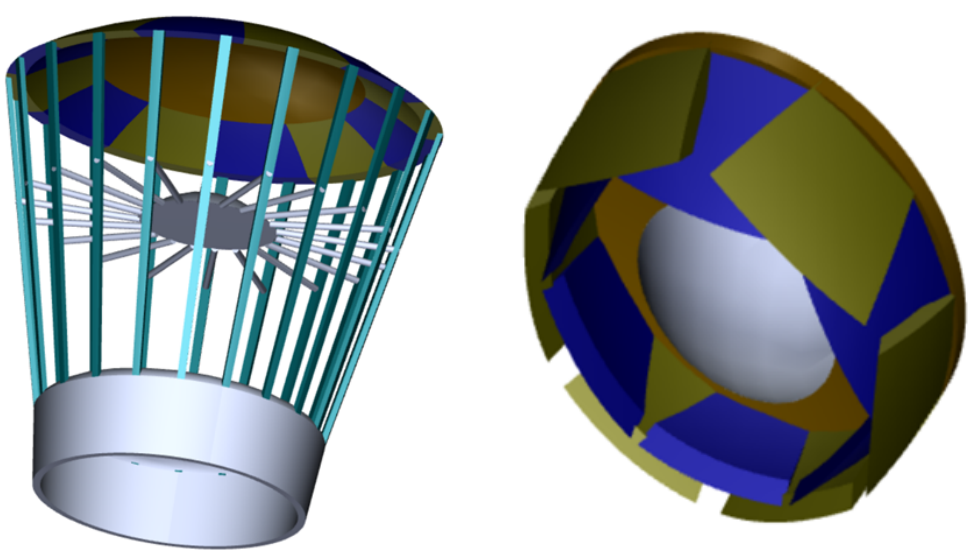

Figure 6: 3D model of K-EUSO mechanical structure. Left: in operation mode. Right: during transportation

\section{Conclusions}

The K-EUSO experiment is a joint effort of SINP MSU and the JEM-EUSO collaboration to realize a large EECR detector on board the Russian segmemt of the ISS based on the reflecting optical system modified using the experience in the collaboration. Project has been accepted to the Long Term Program of Applied Scientific Research and Space Experiments on the ISS in 2010 and it has passed a preliminary feasibility study in 2012 .

A new Schmidt type telescope was developed to enlarge the detector FOV up to $40^{\circ}$. The new logic architecture of the focal surface is proposed using a new generation of system-on-chip FPGA. Now the project undergoes the Design Project stage, accepted to the Stage program of experiments on board the RS of the ISS and planned for launch in 2022.

\section{Acknowledgement}

This work was partially supported by State Space Corporation ROSCOSMOS, the Russian Foundation for Basic Research, grant \#16-29-13065. 


\section{References}

[1] J. Linsley, L. Scarsi and B. Rossi, Extremely Energetic Cosmic-Ray Event, Physical Review Letters 6 (May, 1961) 485-487.

[2] Pierre Auger collaboration, A. Aab et al., Searches for Anisotropies in the Arrival Directions of the Highest Energy Cosmic Rays Detected by the Pierre Auger Observatory, Astrophys. J. 804 (2015) 15, [1411.6111].

[3] Telescope ARray collaboration, R. U. Abbasi et al., Indications of Intermediate-Scale Anisotropy of Cosmic Rays with Energy Greater Than 57 EeV in the Northern Sky Measured with the Surface Detector of the Telescope Array Experiment, Astrophys. J. 790 (2014) L21, [1 404.5890 ].

[4] R. Benson and J. Linsley, Satellite observation of cosmic ray air showers, in 17th International Cosmic Ray Conference, Paris, France, vol. 8, pp. 145-148, 1981.

[5] V. Abrashkin, V. Alexandrov, Y. Arakcheev, E. Bitkin, A. Cordero, S. Eremin et al., The TUS space fluorescence detector for study of UHECR and other phenomena of variable fluorescence light in the atmosphere, Advances in Space Research 37 (2006) 1876-1883.

[6] J. H. Adams, S. Ahmad, J.-N. Albert, D. Allard, L. Anchordoqui, V. Andreev et al., Space experiment TUS on board the Lomonosov satellite as pathfinder of JEM-EUSO, Experimental Astronomy 40 (Nov., 2015) 315-326.

[7] B. A. Khrenov, V. V. Alexandrov, D. I. Bugrov, G. K. Garipov, N. N. Kalmykov, M. I. Panasyuk et al., KLYPVE/TUS space experiments for study of ultrahigh-energy cosmic rays, Physics of Atomic Nuclei 67 (Nov., 2004) 2058-2061.

[8] G. K. Garipov, V. V. Alexandrov, D. I. Bugrov, A. Cordero, M. Cuautle, B. A. Khrenov et al., Electronics for the KLYPVE Detector, in Observing Ultrahigh Energy Cosmic Rays from Space and Earth (H. Salazar, L. Villasenor and A. Zepeda, eds.), vol. 566 of American Institute of Physics Conference Series, pp. 76-90, May, 2001, DOI.

[9] M. Panasyuk, P. Klimov, B. Khrenov, S. Sharakin, M. Zotov, P. Picozza et al., Ultra high energy cosmic ray detector KLYPVE on board the Russian Segment of the ISS, in 34th International Cosmic Ray Conference (ICRC2015), vol. 34 of International Cosmic Ray Conference, p. 669, July, 2015.

[10] M. I. Panasyuk, M. Casolino, G. K. Garipov, T. Ebisuzaki, P. Gorodetzky, B. A. Khrenov et al., The current status of orbital experiments for UHECR studies, in Journal of Physics Conference Series, vol. 632 of Journal of Physics Conference Series, p. 012097, Aug., 2015, 1501.06368 , DOI.

[11] THE JEM-EUSO collaboration, M. Casolino et al., KLYPVE-EUSO: Science and UHECR observational capabilities, in 35th International Cosmic Ray Conference, 2017.

[12] THE JEM-EUSO collaboration, P. Sandri et al., Schmidt type optical system for the KLYPVE-EUSO UHECR detector, in 35th International Cosmic Ray Conference, 2017.

[13] J. H. Adams, S. Ahmad, J.-N. Albert, D. Allard, M. Ambrosio, L. Anchordoqui et al., An evaluation of the exposure in nadir observation of the JEM-EUSO mission, Astroparticle Physics 44 (Apr., 2013) 76-90, [1305.2478].

[14] THE JEM-EUSO collaboration, A. Belov et al., Mini-EUSO photodetector module data processing system, in 35th International Cosmic Ray Conference, 2017. 